\title{
Antibiotic Resistance in Children with Recurrent or Complicated Urinary Tract Infection
}

\author{
Younis N,' Quol K, ${ }^{2}$ Al-Momani T,' Al-Awaisheh F,' Al-Kayed D² \\ 'Department of Pediatric, Prince Hashem Hospital, ${ }^{2}$ Department of Pharmacology and Supply, Prince Hashem Hospital
}

\section{ABSTRACT}

Introduction: Urinary tract infection is certainly one of the most common childhood infections. Emerging resistance to the antibiotics is not unusual. Current hospitalization for children with urinary tract infection is reserved for severe or complicated cases. The aim of the present study was to determine the antibiotic resistance pattern among children with recurrent or complicated urinary tract infection.

Methods: A retrospective study carried out at Prince Hashem hospital, Zarqa city, eastern Jordan and involved 336 episodes of culture proved urinary tract infection obtained from 121 patients with recurrent UTI, who used prophylactic antibiotics during the period from April 1, 2004 to December 31, 2006. The isolated microorganisms and there antibiotics susceptibility were studied.

Results: Seventy three patients $(60.3 \%)$ were found to have some forms of urinary tract anomaly, significantly more prevalent among male children $\mathrm{P}<0.001$. Vesicoureteral reflux being the most common (58.9\%). Renal scars were significantly more prevalent among those with complicated rather than recurrent urinary tract infection $(64.3 \%$ vs. $16.6 \%, \mathrm{P}<0.001)$. Gram negative organisms were the most frequent isolates in patients with recurrent and complicated urinary tract infection. Proteus, Pseudomonas and Candida spp. were more prevalent in patients with complicated $(\mathrm{P}<0.001)$, and isolates in patients with UTA were significantly more resistant to most antibiotics tested.

Conclusions: Pediatric urine culture isolates are becoming increasingly resistant to commonly used antibiotics. Empirical treatment with Trimethoprim-Sulfamethoxazole (TMP-SMX) or Cephalexin as the initial drug is ineffective. Nitrofurantoin and Nalidixic acid can be considered as the first line antibiotics for prophylaxis and or treatment of patients with recurrent UTI, while Meropenam and Ciprofloxacin can be used empirically in treating patients with complicated UTI.

Key words: Antibiotic resistance, Complicated, Recurrent, Urinary tract infection

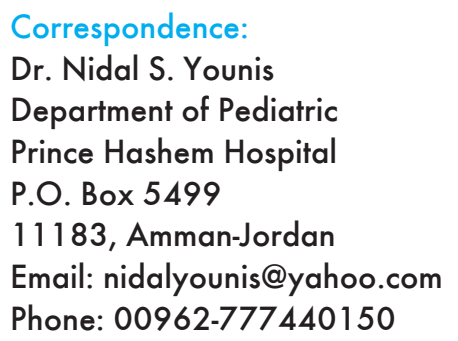




\section{INTRODUCTION}

Urinary tract infection (UTI) is one of the most common bacterial diseases in children; it is acquired by an estimated $3-5 \%$ of girls and $1 \%$ of boys. ${ }^{1-3}$ The true incidence of UTI in children is difficult to estimate, particularly because young children with UTI may only have fever and no specific urinary tract symptoms or signs. ${ }^{1}$ The prognosis is usually favorable, but it relies on timely administration of appropriate antimicrobial treatment. ${ }^{1,2}$

Most urinary tract infections are uncomplicated and respond readily to treatment. ${ }^{4-6}$ However, $30-40 \%$ of these patients have another episode within 2 years, particularly in girls. Also, recurrent infections may be complicated..$^{4,7}$ Treatment for recurrences or complicated UTI is difficult and serious sequel may be seen. ${ }^{7}$

In patients with suspected UTI, antibiotic treatment is usually started empirically. To insure appropriate treatment, knowledge of the organisms that cause UTI and their antibiotic susceptibility is mandatory to eliminate the symptoms, eradicate the infection, prevent urosepsis and to reduce the likelihood of renal damage..$^{2,5,8,9}$

The aim of this study was to delineate the uropathogens recovered in children with recurrent or complicated urinary tract infection, to study the pattern of antibiotic susceptibility and to recommend initial empiric antibiotic prescription before the result of urine culture become available.

\section{METHODS}

A retrospective study carried out at Pediatric department, Prince Hashem general hospital, located in Zarqa city at eastern part of Jordan, and involved 121 patients with recurrent UTI, who were treated as either inpatients or at outpatient's clinics during the period between April 1, 2004 and December 31, 2006.

Patients with recurrent UTI were separated into 2 groups: Group 1 for patients with renal anomaly and group 2 for those without renal anomaly. All these patients received antimicrobial prophylaxis against UTI.

Cultures were obtained by suprapubic aspiration, transurethral bladder catheterization or midstreamcollected urine; bag-collected urine cultures were not included. Identification of microbial growth and determination of antimicrobial susceptibility done by the disk diffusion technique, with the recommended media and standard control strains. ${ }^{10}$ Susceptibility was routinely tested for the following antimicrobial agents: Ampicillin, Ceftriaxone, Cephalexin, Ciprofloxacillin, Gentamicin, Meropenam, Nalidixic acid, Nitrofurantoin, Norfloxacin and Trimethoprim-Sulfamethoxazole.
UTI was defined according to the AAP guidelines. ${ }^{8}$ Cultures were considered positive when there was growth of a single pathogen of $>10^{5}$ colony forming units $/ \mathrm{ml}$ in a urine specimen collected by midstream catch; of $>10^{4}$ colony forming units $/ \mathrm{ml}$ in urine collected by bladder catheterization; and any growth in urine obtained by a suprapubic aspiration. Mixed pathogen growth results were considered unreliable and been excluded.

Recurrent UTI was defined as a single further infection by a new organism. ${ }^{7}$ The UTI occurring in the presence of catheterization, functional or anatomical abnormalities of the of the urinary tract, host with altered defenses, chronic renal failure, renal transplantation, and those receiving peritoneal and hemodialysis were defined as complicated UTI. ${ }^{11}$

Abdominal ultrasound, technetium 99m dimercaptosuccinic acid (DMSA) scan and voiding cystourethrogram were performed in all cases. Diethylene traimine pentaacetic acid (DTPA) was performed in children with the obstructive anomaly. The DMSA scan was routinely done at the time of diagnosis and at the follow up after six months in patients with renal scars.

Patients were divided into two groups: Group 1 for patients with renal anomaly, and group 2 for those without renal anomaly. All these patients received antimicrobial prophylaxis against UTI. Both groups were compared for gender deference and antimicrobial susceptibility.

Statistical Package for Social Sciences for windows, version 10 was used for statistical analysis. The Chi-Square as well as Fisher's exact test were used to compare categoric variables. $\mathrm{P}$ value $<0.05$ was considered significant.

\section{RESULTS}

Three hundred thirty six episodes of culture proved UTI obtained from 121 patients with recurrent UTI were enrolled in the study; 28 boys $(23.2 \%)$ and 93 girls $(76.8 \%)$. Mean age was $6.8 \pm 4.1$ years (range $1-14$ years) (Table 1,2).

Seventy three patients (60.3\%) had some forms of UTA (Table 3). Renal scars were seen in 47 patients with UTA $(64.3 \%)$ and in 8 patients without UTA (16.6\%). Renal scars formation in patients with vesicoureteral reflux (VUR) was seen in 34 patients $(79.1 \%)$ and in 21 patients $(26.9 \%)$ without VUR $(P<0.001)$.

Escherichia coli (E. coli) was determined to be the predominant microorganism in both groups; it was seen in 104 patients (71\%) with recurrent UTI, and in 91 patients (47\%) with complicated UTI. Proteus, 
Pseudomonas and Candida spp. were significantly more prevalent in patients with complicated UTI $(\mathrm{P}<0.001)$ (Table 4).

Susceptibility was tested; the overall resistance to antibiotics was as follow: Ampicillin 73\%, Ceftriaxon $11.8 \%$, Cephalexin 41.1\%, Ciprofloxacillin 3.8\%, Gentamicin 43.2\%, Meropenam 2\%, Nalidixic acid 29.3\%, Nitrofurantoin 13.3\%, Norfloxacin 11.0\% and Trimethoprim-Sulfamethoxazole $59.5 \%$ as shown in (Figure 1). Total antibiotic resistance ratios for Ceftriaxon, Gentamicin, Nalidixic acid and Nitrofurantoin, were significantly more common in patients with complicated UTI (Table 5).

\section{DISCUSSION}

The UTI has a tendency to recur in children with renal anomaly or preexisting renal disease, and its importance, as a cause of renal insufficiency is well known. There are multiple risk factors for the UTI in pediatric patients, including age, gender, periurethral or colonization factors, native immunity, genitourinary abnormalities, genetic, and iatrogenic factors. ${ }^{12}$ Patients with recurrent UTI who have such risk factors, are at increased risk of pyelonephritis and subsequent risk of renal scaring with progressive renal disease in adulthood. ${ }^{13}$

The incidence of VUR is $1-3 \%$ among children, and 20 $75 \%$ of them have UTI. ${ }^{8,14}$ In our study the prevalence rate was $58.9 \%$ (Table 3 ), and VUR was found to be the most common anatomic malformation in patients with complicated UTI. Renal scaring was clamed to be related to the presence of anatomic malformation. ${ }^{15}$ Our data support this theory; renal scars were seen in $79.1 \%$ of patients with VUR, while it was $24.3 \%$ of patients without VUR $(P<0.05)$. As well, the prevalence rate of renal scare was also high in patients with other anomalies (43.3\%).

According to demographic data, females are affected more often than males, but the prevalence of resistant microorganisms was less among females than males for all agents under study. This trend is explained by the tendency of males to present more often with complicated UTIs (75\%), which may be associated with more antimicrobial resistant pathogens. Similar results were reported by Prais ${ }^{3}$ and Howard. ${ }^{16}$
Enterobacteriaceae are the most common organisms isolated from patients with UTI. Of these, E. coli account for $65-90 \%$ of all urinary infections in children. ${ }^{1,3,5}$ In our study, the overall prevalence rate for $\mathrm{E}$ - coli was $58 \%$, but among patients with recurrent UTI, it was $71.7 \%$. While among patients with complicated UTI; Proteus, Pseudomonas, and Candida spp. were significantly more prevalent $(\mathrm{P}<0.001)$, and this results might be explained by the misuse or the abuse of broad spectrum antibiotics, as well as, the frequent hospitalization of patients with complicated UTI (Table 4).

The most common empiric oral antibiotics prescribed in our practice for treatment and for secondary prophylaxis of UTI are first generation Cephalosporins (Cephlexin), and Trimethoprim- Sulfamethoxazole (TMP-SMX). Our study shows an increase bacterial resistance to commonly used oral antibiotics in children with UTI and particularly to the above mentioned drugs; it was $41 \%$ and $59 \%$ respectively. Raz in his study on children with UTI aged 1-15 years reported even a higher percentage of uropathogen resistant to Ampicillin and Cephalexin with slightly less resistance to Trimethoprim-Sulfamethoxazole (TMP-SMX). ${ }^{17}$ They were $88 \%, 44 \%$ and $52 \%$ respectively.

In 1999, Hoberman and colleagues recommended oral Cefixime for the treatment of young children with fever and UTI. Cefixime was not tested in our study, but the published data support the efficacy of this third generation cephalosporin to be used empirically. ${ }^{6}$

Among bacterial isolates from children with UTI in our study, resistance to Meropenem and Ciprofloxacin were the least. It was $1.7 \%$ and $3.8 \%$ respectively. This might be due to the limited usage of these antibiotics in our practice. The other two oral antimicrobials which still have relatively low resistance are Nitrofurantoin and Nalidexic acid. Nitrofurantoin is considered one of the oldest urinary anti-infective drugs in use, surprisingly; resistance to this drug remains minimal. As seen in (Table 5), the overall resistance was $13.3 \%$, but among patients with recurrent UTI, the percentage was even less $5.5 \%$. The lack of resistance may be related to the fact that Nitrofurantoin has multiple mechanisms of action, requiring organisms to develop more than a single mutation in order to develop resistance. In

\section{Table 1. Demographic data of the study population}

\begin{tabular}{lccccc}
\hline Age group & \multicolumn{2}{c}{ Female } & \multicolumn{2}{c}{ Male } & Total \\
& Complicated & Recurrent & Complicated & Recurrent \\
\hline 0-1 year & 8 & 1 & 3 & 0 & 12 \\
$1-5$ years & 19 & 11 & 7 & 2 & 39 \\
$>5$ years & 25 & 2 & 11 & 5 & 70 \\
Total & $\mathbf{5 2}$ & $\mathbf{4 1}$ & $\mathbf{2 1}$ & $\mathbf{7}$ & $\mathbf{1 2 1}$ \\
\hline
\end{tabular}


Table 2. Method collection of urine specimen

\begin{tabular}{lc}
\hline Method & Number \\
\hline Mid Stream Urine & 249 \\
Catheter & 39 \\
Suprapubic aspiration & 48 \\
\hline
\end{tabular}

Table 3. Urinary tract anomalies (UTA) among patients with complicated UTI

\begin{tabular}{lcccc}
\hline Urinary anomaly & Male & Female & Total & $\%$ \\
\hline Vesicoureteral reflux & 18 & 25 & 43 & 58.9 \\
Neurogenic bladder & 7 & 14 & 21 & 28.7 \\
Ureteropelvic junction obstruction & 4 & - & 3 & 5.4 \\
Renal hypoplasia & 2 & - & 1 & 4.1 \\
Cystic disease of kidney & 1 & - & 1 & 1.4 \\
Absence of kidney & 1 & 1.4 \\
\hline
\end{tabular}

Table 4. Prevalence of microorganisms in urine cultures

\begin{tabular}{lcccc}
\hline \multicolumn{1}{c}{ Microorganism } & $\begin{array}{c}\text { Complicated } \\
\text { UTI } \\
(\mathbf{N}=191)\end{array}$ & $\begin{array}{c}\text { Recurrent } \\
\text { UTI } \\
\text { (N=145) }\end{array}$ & $\begin{array}{c}\text { Total } \\
(\mathbf{N}=336)\end{array}$ & P value \\
\hline Acinobacter spp. & $4(2.1 \%)$ & $1(0.7 \%)$ & $5(1.5 \%)$ & $>0.05$ \\
Candida spp. & $9(4.7 \%)$ & 0 & $9(2.6 \%)$ & $<0.001$ \\
Citrobacter spp. & $2(1.0 \%)$ & $1(0.7 \%)$ & $3(0.9 \%)$ & $>0.05$ \\
Coagulase Negative & $5(2.6 \%)$ & $3(2.1 \%)$ & $8(2.4 \%)$ & $>0.05$ \\
Staphylococcus & $10(5.2 \%)$ & $6(4.1 \%)$ & $16(4.8 \%)$ & $>0.05$ \\
Enterococcus & $91(47.6 \%)$ & $104(71.7 \%)$ & $195(58 \%)$ & $<0.001$ \\
Escherichia coli & $30(15.7 \%)$ & $21(14.5 \%)$ & $51(15.2 \%)$ & $>0.05$ \\
Klebsiella spp. & $25(13.1 \%)$ & $8(5.5 \%)$ & $33(9.8 \%)$ & $<0.001$ \\
Proteous & $14(7.3 \%)$ & $1(0.7 \%)$ & $15(4.5 \%)$ & $<0.001$ \\
Pseudomonus & $1(0.5 \%)$ & 0 & $1(0.3 \%)$ & $>0.05$ \\
Staphylococcus aureus & & & & \\
\hline
\end{tabular}

Table 5. Antibiotic resistances among all isolates

\begin{tabular}{lcccc}
\hline & \multicolumn{2}{c}{ Resistance } & & \\
Antimicrobial & Recurrent UTI & Complicated UTI & All pathogens & $\mathbf{P}$ \\
& $\mathbf{n . 1 4 5}$ & $\mathbf{n . 1 9 1}$ & $\mathbf{n . 3 3 6}$ & value \\
\hline Ampicillin & $\mathbf{( 4 3 . 1 \% )}$ & $\mathbf{( 5 6 . 9 \% )}$ & & $>0.05$ \\
Ceftriaxon & $104(\mathbf{7 1 . 7 \% )}$ & $142(74.3 \%)$ & $246(73.3 \%)$ & 0.0001 \\
Cephalexin & $11(7.5 \%)$ & $28(14.6 \%)$ & $39(11.8 \%)$ & $>0.05$ \\
Ciprofloxacillin & $57(39.3 \%)$ & $81(42.4 \%)$ & $138(41.1 \%)$ & $>0.05$ \\
Gentamicin & $5(3.4 \%)$ & $8(4.2 \%)$ & $13(3.8 \%)$ & 0.0001 \\
Meropenem & $42(28.9 \%)$ & $103(53.9 \%)$ & $145(43.2 \%)$ & $>0.05$ \\
Nalidixic acid & $2(1.3 \%)$ & $4(2.0 \%)$ & $6(1.7 \%)$ & 0.0001 \\
Nitrofurantoin & $31(21.3 \%)$ & $67(35.0 \%)$ & $98(29.3 \%)$ & 0.0001 \\
Norfluxacin & $8(5.5 \%)$ & $37(19.3 \%)$ & $45(13.3 \%)$ & $>0.05$ \\
Trimethoprim- Sulfamethoxazole & $15(10.3 \%)$ & $22(11.5 \%)$ & $37(11.0 \%)$ & $>0.05$ \\
\hline
\end{tabular}




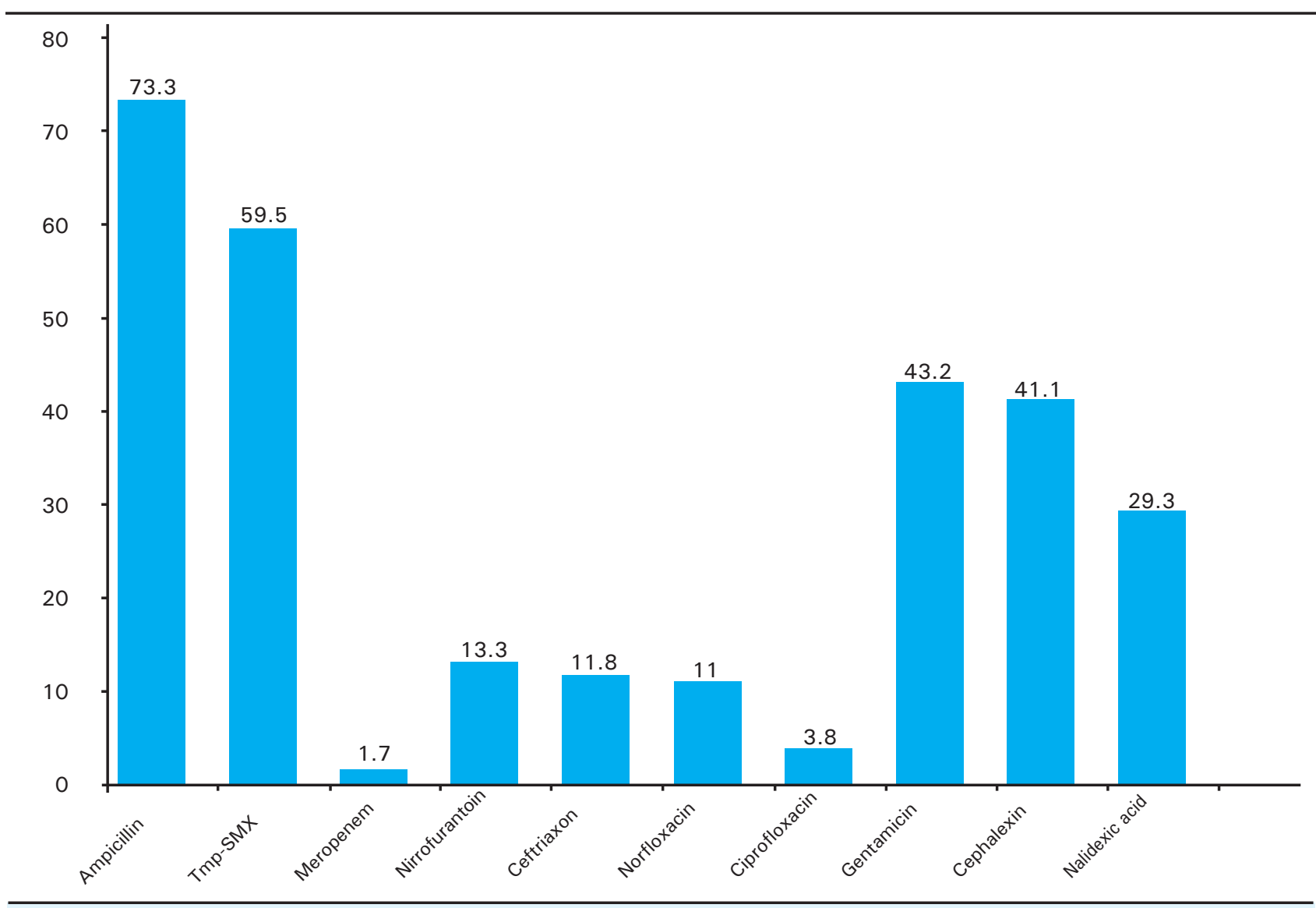

Figure 1. Antibiotics resistance among urinary isolates

addition, limited usage of Nitrofurantoin for treating uncomplicated cystitis, may also be a contributing factor to the lack of development of widespread resistance to this drug. ${ }^{18,19}$ The other oral antimicrobial agent who maintains to a less degree a high efficacy against urinary pathogens is Nalidixic acid. The overall resistance in our study was $29.3 \%$, while among patients with recurrent UTI, it was $21.3 \%$. Even more encouraging results regarding these antimicrobials were reported by Prais $^{3}$ which makes these drugs a good option for initial empirical treatment of UTI in children.

\section{CONCLUSIONS}

Pediatric urine culture isolates are becoming increasingly resistant to commonly used antibiotics. Empirical treatment with Trimethoprim-Sulfamethoxazole (TMPSMX) or Cephalexin as the initial drug is ineffective. Nitrofurantoin and Nalidixic acid can be considered as the first line antibiotics for prophylaxis and or treatment of patients with recurrent UTI, while Meropenem and Ciprofloxacin can be used empirically in treating patients with complicated UTI.

\section{REFERENCES}

1. Schlager TA. Urinary tract infections in infants and children. Infect Dis Clin North Am 2003;17:353-365.

2. Elder JS. Urologic disorders in infants and children. In: Behrman RE, Kliegman RM, Jenson HB, eds. Nelson Textbook of Pediatrics. 17th ed. Philadelphia, PA: W. B. Saunders; 2004. p.1785-1790.

3. Prais D, Straussberg R, Avitzur Y, et al. Bacterial susceptibility to oral antibiotics in community acquired urinary tract infection. Arch Dis Child 2003;88:215-218.

4. Hoberman A, Han-Pu C, Keller DM, et al. Prevalence of urinary tract infection in febrile infants. J Pediatr 1993;123:723.
5. Ashkenazi S, Even-Tov S, Samra Z, et al. Uropathogens of various childhood populations and their antibiotic susceptibility. Pediatr Infect Dis J 1991;10:742-746.

6. Hoberman A, Wald ER, Kickey RW, et al. Oral versus initial intravenous therapy for urinary tract infections in young febrile children. Pediatrics 1999;104:79-86.

7. Larcombe J. Urinary tract infection in children. Clin Evid 2005;429-440.

8. Committee on Quality Improvement. Subcommittee on Urinary Tract Infections. Practice parameter: the diagnosis, treatment, and evaluation of the initial UTI in febrile infants and young children. Pediatrics 1999;103:843-852. 
9. Gaspari RJ, Dickson E, Karlowsky J, Doern G. Multidrug resistance in pediatric urinary tract infections. Microb Drug Resist 2006;12:126-129.

10. National Committee for Clinical Laboratory Standards. Performance standards for antimicrobial susceptibility testing. Fourth Informational Supplement. NCCLS Document M7-A3. Villanova, PA: Clinical and Laboratory Standards Institute, 1994.

11. Melekos MD, Naber KG. Complicated urinary tract infections. Int J Antimicrob Agents 2000;15:247-256.

12. Chang SL, Shortliffe LD. Pediatric urinary tract infections. Pediatr Clin North Am 2006; 53:379-400

13. Wennerstorm M, Hansson S, Jodal U, Stokland E. Primary and acquired renal scaring in boys and girls with urinary tract infection. J Pediatr 2000;136:2-4

14. Blumenthal I. Vesicoureteral reflux and urinary tract infection in children. Postgrad Med J 2006;82:31-35
15. Orellana P, Baquedano P, Rangarajan V, Zhao JH, Eng ND, Fettich J, et al. Relationship between acute pyelonephritis, renal scaring, and Vesicoureteral reflux. Results of a coordinated research project. Pediatr Nephrol 2004;19:11221126.

16. Howard AJ, Magee JT, Fitzgerald KA, Dunstan FDJ. Factors associated with antibiotic resistance in coliform organisms from community urinary tract infection in Wales. J Antimicrob Chemother 2001;47:305-313.

17. Raz R, Kov N, Kennes Y, et al. Demographic characteristics of patients with community-acquired bacteriuria and susceptibility of urinary pathogens to antimicrobials in northern Israel. Isr Med Assoc J 2000;2:426-9

18. Ladhani S, Gransden W. Increasing antibiotic resistance among urinary tract isolates. Arch Dis Child 2003;88:444-445

19. Gupta K. Emerging antibiotic resistance in urinary tract pathogens. Infect Dis Clin North Am 2003;17:243-259. 